Received 08/10/2017

Review began 08/29/2017

Review ended 09/11/2017

Published 09/12/2017

C Copyright 2017

Busu et al. This is an open access article distributed under the terms of the Creative Commons Attribution License CC-BY 3.0., which permits unrestricted use, distribution, and reproduction in any medium, provided the original author and source are credited.

\title{
Iatrogenic Circumflex Artery Stenosis Following Mitral Valve Repair
}

\author{
Tatiana Busu ${ }^{1}$, Fahad Alqahtani ${ }^{2}$, Akram Kawsara ${ }^{2}$, Mohamad Hijazi ${ }^{1}$, Mohamad Alkhouli ${ }^{2}$ \\ 1. Heart and Vascular Institute, West Virginia University School of Medicine/Ruby Memorial Hospital 2. \\ Division of Cardiovascular Disease, West Virginia University School of Medicine/Ruby Memorial Hospital
}

$\square$ Corresponding author: Tatiana Busu, tatianabusu@gmail.com

Disclosures can be found in Additional Information at the end of the article

\section{Abstract}

Injury of the left circumflex coronary artery is a potentially serious complication of mitral valve surgery due to the proximity of the vessel to the posterior segment of the mitral annulus. Suture-related distortion of the artery with partial or subtotal occlusion is the most commonly implicated mechanism. Herein, we present a case of symptomatic iatrogenic circumflex coronary artery stenosis following mitral valve annuloplasty for degenerative mitral valve regurgitation.

Categories: Cardiac/Thoracic/Vascular Surgery, Cardiology, Internal Medicine

Keywords: mitral valve repair, iatrogenic injury, left circumflex, optical coherence tomography

\section{Introduction}

An iatrogenic coronary artery injury following cardiac valve surgery is a rare but underrecognized entity. The proximity of the left circumflex coronary artery to the mitral annulus makes this artery particularly susceptible to hemorrhagic and nonhemorrhagic injuries during mitral valve repair and replacement surgeries [1]. An unintentional capture of the artery with the suture material during surgery can lead to an iatrogenic coronary stenosis. The recognition of this complication can be difficult due to the variable manifestation and the lower threshold of subject patients with previously normal coronaries to further testing [2]. We present a case of an undiagnosed iatrogenic left circumflex coronary artery following mitral valve repair that manifested with recurrent effort angina. In this manuscript, we aim to raise awareness of this rare but potentially debilitating complication.

\section{Case Presentation}

A 67-year-old male presented with recurrent exertional chest pain. The patient has a history of atrial fibrillation and severe degenerative mitral regurgitation but no history of coronary artery disease. Two years prior, he underwent mitral valve repair with a quadrangular resection of the posterior leaflet and ring annuloplasty with a 34-mm Memo 3D annuloplasty ring (Sorin Group, Italy), a modified maze procedure, and an exclusion of the left atrial appendage with AtriCure (AtriCure, Mason, OH). His preoperative angiogram showed no significant coronary disease (Figure 1). 


\section{Cureus}

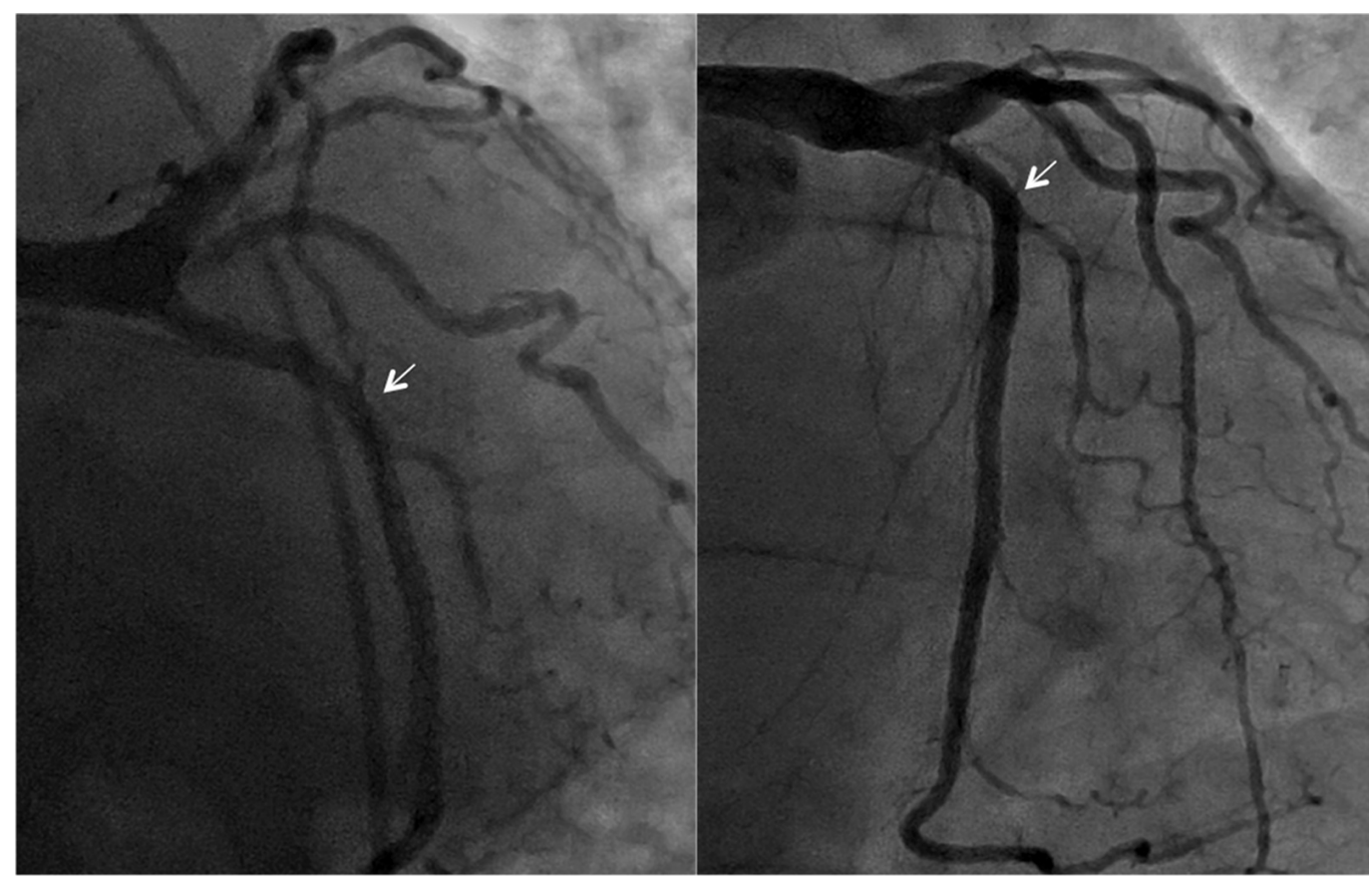

\section{FIGURE 1: Preoperative coronary angiography showing no obstructive disease in the left circumflex coronary artery}

Due to his recurrent chest pain, a repeat angiogram was done, which showed severe stenosis and distortion of the mid circumflex artery in close proximity to the suture line of the mitral ring (Figure 2A). The lesion was crossed with a 0.014 " Runthrough wire with moderate difficulty (Terumo, Somerset, NJ). Optical coherence tomography (OCT) was then performed with a Dragonfly OPTIS imaging catheter (St. Jude Medical, Minnesota, United States) and showed no significant atherosclerosis in the circumflex artery but a severe iatrogenic stenosis of the mid segment of the artery (Figure 2A'). The lesion was dilated with a 2.5x12 mm Sprinter RX balloon (Medtronic, Minnesota, United States) and stented with a 3.5x15 mm Xience stent (Abbott Vascular, Santa Clara, CA) (Figure 2B). Post-stenting angiography and OCT imaging demonstrated complete resolution of the stenosis and a well-apposed stent (Figure 2B').

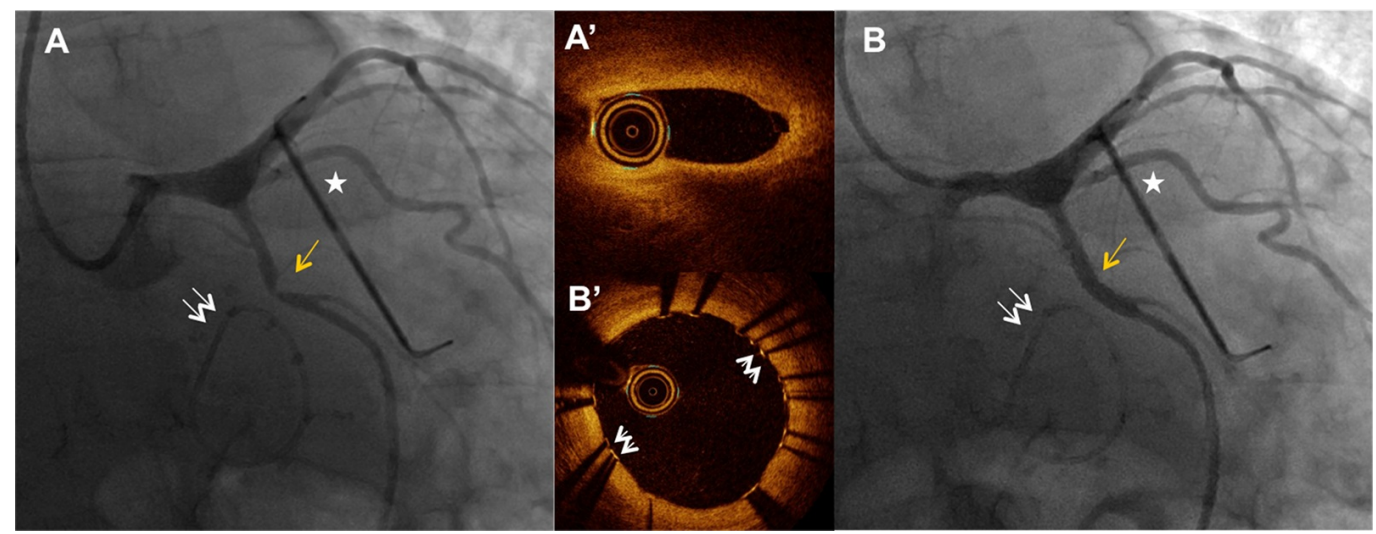

FIGURE 2: latrogenic circumflex stenosis adjacent to the mitral ring

2A - Postsurgery coronary angiography showing severe mid circumflex stenosis (yellow arrow). Double arrows indicate the mitral ring and the star indicates the AtriCure left atrial appendage occlusion device. 
$2 A^{\prime}$ - Optical coherence tomography showing the site of iatrogenic stenosis.

2B - Post-balloon angioplasty and stenting angiography showing resolution of the stenosis.

2B' - Optical coherence tomography after stenting showing a well-apposed stent and no residual stenosis. The white arrows annotate the stent struts.

Following the intervention, the patient's chest pain has resolved and he remained free from chest pain at the four weeks follow-up.

\section{Discussion}

Iatrogenic coronary artery stenosis is a rare complication of mitral valve surgery due to the close proximity of the circumflex artery to the mitral valve annulus [3-4]. Various underlying causes of injury have been proposed; laceration of the artery can lead to both hemorrhagic complications as well as a thrombotic occlusion due to an injury of the endothelium, both with a potentially acute onset of symptoms. A more insidious onset of symptoms usually occurs with suture fixation with either complete encircling with occlusion of the artery or distortion of vessel anatomy due to suture fixation, or due to a dynamic occlusion secondary to spasm and/or subintimal hematoma [5]. Among all these potential causes, a suture-related injury is considered the most common etiology of iatrogenic coronary injuries [4].

The underlying etiology can be often suggested with coronary angiography. However, intravascular imaging with intravascular ultrasound or OCT can bring further anatomical details and aid in the ascertainment of that etiology. In this case, OCT revealed the absence of atherosclerosis, intimal or subintimal injury, and vessel wall hematoma and confirmed that the likely etiology was a suture-related encircling of the vessel. In this case, percutaneous balloon angioplasty and stenting were feasible and resulted in immediate symptomatic relief.

\section{Conclusions}

The close anatomical proximity of the circumflex coronary artery to the posterior mitral annulus presents a risk of an iatrogenic injury of the vessel during surgical mitral valve repair or replacement. Increased awareness of this potential complication can aid in early identification and effective treatment.

\section{Additional Information \\ Disclosures}

Human subjects: Consent was obtained by all participants in this study. Conflicts of interest: In compliance with the ICMJE uniform disclosure form, all authors declare the following: Payment/services info: All authors have declared that no financial support was received from any organization for the submitted work. Financial relationships: All authors have declared that they have no financial relationships at present or within the previous three years with any organizations that might have an interest in the submitted work. Other relationships: All authors have declared that there are no other relationships or activities that could appear to have influenced the submitted work.

\section{References}

1. Pessa CJN, Gomes WJ, Catani R, Prates JC, Buffolo E: Anatomical relationship between the posterior mitral valve annulus and the coronary arteries. Implications to operative treatment. 


\section{Cureus}

Braz J Cardiovasc Surg. 2004, 19:372-377.

2. Ziadi J, Mleyhi S, Denguir R, Khayati A: Iatrogenic occlusion of the circumflex artery and left ventricle pseudoaneurysm after mitral annuloplasty. JC Cases. 2014, 9:104-105.

10.1016/j.jccase.2013.10.013

3. Somekh NN, Haider A, Makaryus AN, Katz S, Bello S, Hartman A: Left circumflex coronary artery occlusion after mitral valve annuloplasty: "a stitch in time". Tex Heart Inst J. 2012, 39:104-107.

4. Hiltrop N, Bennett J, Desmet W: Circumflex coronary artery injury after mitral valve surgery: a report of four cases and comprehensive review of the literature. Catheter Cardiovasc Interv. 2017, 89:78-92. 10.1002/ccd.26449

5. Grande AM, Fiore A, Massetti M, Vigano M: Iatrogenic circumflex coronary lesion in mitral valve surgery. Tex Heart Inst J. 2008, 35:179-183. 